\title{
Integrating episodic memories and prior knowledge at multiple levels of abstraction
}

\author{
Pernille Hemmer and Mark Steyvers \\ University of California, Irvine, California
}

\begin{abstract}
Prior knowledge can have a large influence on recall when the memory for the original event is error prone or incomplete. We investigated the interaction between memory and prior knowledge in a recall task involving natural objects such as fruits and vegetables. We first quantified prior knowledge for the sizes of objects in a norming experiment. We then assessed the influence of prior knowledge in a memory experiment in which we compared the actual size of objects shown during a study phase with the reconstructed size of an object during the test phase. Recall was biased both by the mean size of the specific object studied and by the mean size of all objects in the category. This result suggests that the influence of prior knowledge can come from multiple, hierarchically related levels of representation, such as the object-category and superordinate-category levels.
\end{abstract}

Reconstructing events from memory involves the coordination of multiple sources of information. Recall of past events, such as the time we went to work or the size of the coffee we ordered last week, might be based partially on vague recollections of the events themselves but also on prior knowledge; perhaps we usually go to work at a specific time and tend to order coffee of a specific size. Such knowledge might provide useful cues when recalling past events. Bartlett's (1932) seminal research on reconstructive memory initiated many research paradigms that demonstrated how cultural and social norms, as well as cognitive expectations, influence our recall of past events. For example, height judgments can be biased by gender (Biernat, 1993), and face recognition can be influenced by ethnicity (Rehnman \& Herlitz, 2006). Sometimes the expectations based on prior knowledge can lead to systematic errors and intrusions in recall. An office without books can later be remembered as having books, presumably because most offices are expected to have books (Brewer \& Treyens, 1981). Similarly, words can be falsely recalled from a list, especially when the list is structured to have strong associations to a word not present on the list (Roediger \& McDermott, 1995).

On the other hand, prior knowledge can also be an aid to memory. Recall for abstract information typically improves when additional meaningful information is available (Bartlett, 1932; Bower, Karlin, \& Dueck, 1975; Bransford \& Johnson, 1972). Prior knowledge can also be advantageous in recall when the episodic memories for the original event are noisy or incomplete. Huttenlocher and colleagues (Crawford, Huttenlocher, \& Engebretson, 2000; Huttenlocher, Hedges, \& Duncan, 1991; Hutten- locher, Hedges, \& Vevea, 2000) presented a model of category effects in which reconstruction from memory is based on a weighted average of episodic memory traces and prior knowledge in the form of category information. By combining both episodic and category information in recall, large fluctuations in reconstructions due to noisy episodic memories can be prevented. The result of this weighted average is that reconstructions are systematically biased toward the category center. Such systematic biases have also been found in memory psychophysics experiments that reveal a compression of estimated stimulus attributes when those attributes are recalled from memory (Kerst \& Howard, 1978; Moyer, Bradley, Sorensen, Whiting, \& Mansfield, 1978).

Previous studies (e.g., Huttenlocher et al., 1991; Huttenlocher et al., 2000) focused on categorization, using relatively short-term memory tasks in which participants were tested immediately after stimulus exposure. With such short lags, retrieval from episodic memory is less error prone, and it might be more difficult to observe the influence of prior knowledge. Previous studies have also relied on estimation of relatively artificial stimuli, such as the locations of dots placed in a circle, the lengths of lines, or the sizes of blue and red circles (e.g., Huttenlocher et al., 1991; Huttenlocher et al., 2000; Sailor \& Antoine, 2005). Participants are unlikely to have strong preexperimental knowledge for such stimuli, and instead, the prior knowledge is created during the course of the experiment by training participants on different stimulus distributions. In addition, some researchers have argued that any central bias can simply be explained by sequential effects (Sailor \& Antoine, 2005). Because the small- 
est stimuli are always preceded by a larger stimulus, and vice versa, temporal contiguity can result in a central bias, and no explanation in terms of prior knowledge is needed.

In this research, we investigated the interaction between prior knowledge and recall, using an experimental paradigm that differs in three important respects from previous studies. First, our memory tasks emphasized memory on a longer time scale, which we expected to lead to a decrease in the accuracy of the episodic memory representations and a concomitant greater reliance on prior knowledge. This allowed us to better investigate the influence of prior knowledge on recall and to emphasize the memory process. Second, unlike in previous studies, we employed naturalistic stimuli, such as fruits and vegetables, for which we expected relatively well-established preexperimental knowledge. We also expected that such naturalistic stimuli would be associated with more structured knowledge representations in which knowledge could be described at several levels of abstraction. In our experiments, we tested memory for one-dimensional stimulus attributesnamely, the size of an object. Prior knowledge for such attributes might exist not only at the superordinate level (e.g., "I expect fruits to be roughly of this size") but also at the object level (e.g., "I expect an apple to be of this size"). Therefore, the inclusion of naturalistic stimuli allowed us to study the influence of prior knowledge at multiple levels of abstraction. Our experiments were furthermore designed to evaluate systematic deviations that are a result of the influences of prior knowledge and not an artifact of sequential dependencies.

In two experiments, we investigated the interaction between prior knowledge and episodic memory. In Experiment 1 , we collected norms for the sizes of natural objects, such as fruits and vegetables. In this norming experiment, we measured the expected sizes of common natural objects. Participants were asked to make judgments both about the mean expected size and about the minimum and maximum expected sizes of the target objects. In Experiment 2 , we measured performance in a reconstructive memory task involving the normed stimuli.

\section{EXPERIMENT 1}

\section{Method}

Participants. Eighteen undergraduate students at the University of California, Irvine, participated in the experiment. They were compensated with course credit.

Materials and Procedure. We sampled 24 high-resolution color images of fruits and vegetables from a large image database (PhotoObject.net). All of the images were photographed against a white background. The fruit and vegetable images had average sizes of $1,126 \times 1,078$ and $1,175 \times 878$ pixels, respectively. These images and a comparison image were presented on two computer screens. Each fruit or vegetable target image was presented on the right screen, along with a slider used to manipulate the size of the object. The comparison image, containing a display of a plate and utensils, was presented on the left screen. Participants were asked to make the following three judgments relative to the comparison image: "What is the average size of an object like this?" "What is the smallest size of an object like this?" and "What is the largest size of an object like this?" Participants resized the image using the slider. Responses were measured on a scale from 0 to 1 , where 0 corresponded to an object scaled to less than 1 pixel on the screen and 1 corresponded to the maximum position of the slider, at which the target object fills either the entire height or width of the screen. The object remained on the screen until the participant was satisfied with the current size judgment and clicked a button to continue. Participants were shown real-life versions of the comparison object (a set of utensils) to ensure agreement on the common size of the object. The images were blocked by category (fruits and vegetables), and the 24 images within each category were presented in random order. The initial size for each image was chosen at random from the following sizes on the slider scale: $.2, .4, .6$, and .8 (proportions of the maximum size of the image).

\section{Results}

Figure 1 shows the mean ratings for 24 objects from the fruit and vegetable categories, ordered by the mean ratings for the average size. ${ }^{1}$ The ordering of sizes reflects the intuitive notions that the participants had about fruits and vegetables. For example, in the fruit category, the average size judgment for a raspberry was smaller than that for a strawberry, which was smaller than that for an apple, all of which in turn were smaller than that for a pineapple. The range of the size judgments also increased with the magnitude of the objects, such that the range for the raspberry was the smallest, and the range for the pineapple was the largest. The participants expressed a reasonable degree of agreement in their judgments. For the average judgment, the mean pairwise rater correlation was .65 , with a $90 \%$ confidence interval of .48 and .81 .

\section{EXPERIMENT 2}

In this experiment, we assessed how prior knowledge for the sizes of objects influences the recalled size of a particular object studied in the context of the experiment. We tested recall for the natural objects that were normed in Experiment 1, as well as for a set of artificial shapes. We predicted that the effect of prior knowledge for fruits and vegetables would occur at two levels of abstraction: the object-category and superordinate-category levels. We hypothesized that recall would be biased toward the mean of the distribution associated with all size variations of the particular object (e.g., an apple) and also toward the mean of the size distribution associated with all objects within the object's superordinate category (e.g., fruits). Therefore, we predicted that an object that is studied at a relatively small or large size relative to the mean of the object would be over- or underestimated, respectively, at test. Similarly, we predicted that an object that is studied at a relatively small or large size relative to the mean of the superordinate category distribution would be over- or underestimated, respectively, at test. These predictions are consistent with the category effects found by Huttenlocher et al. (1991; Huttenlocher et al., 2000), in which small things were made larger at reconstruction, and large things were made smaller at reconstruction. Figure 2 illustrates how the contributions of prior knowledge at the object- and superordinate-knowledge levels can be teased apart. It illustrates the predicted recall for two example objects - a strawberry and an apple — shown at the same 


\section{A}

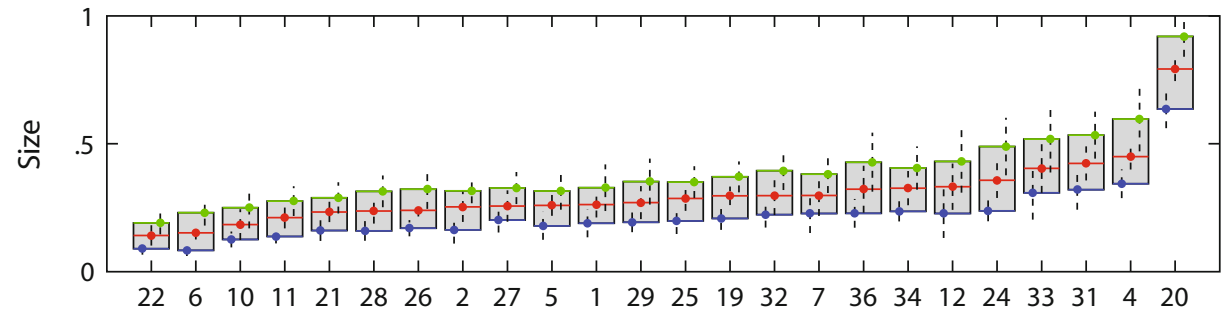

22

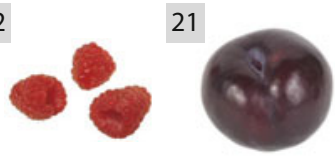

27

25

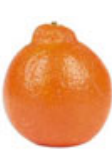

36

33
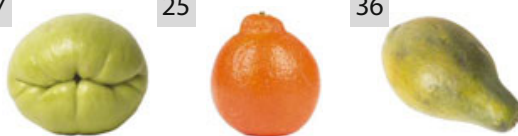

6

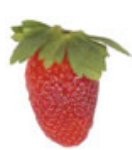

28

5

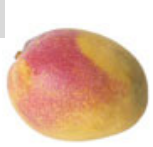

19

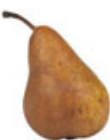

34

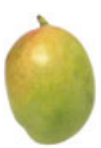

31

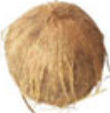

10

26

1

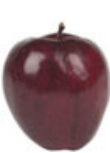

32

12

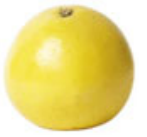

4
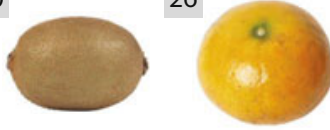

2

29

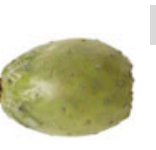

7

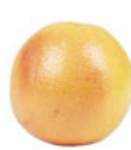

24
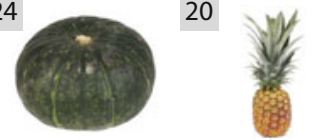

B
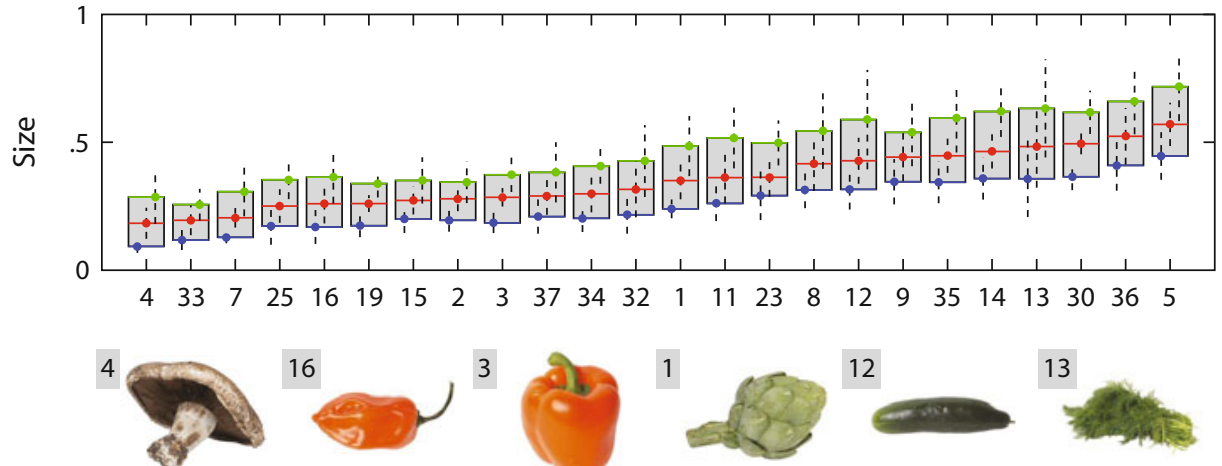

16
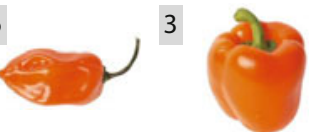

1

12

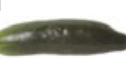

13

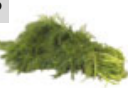

33

19

37

11
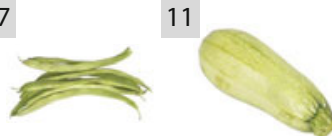

9

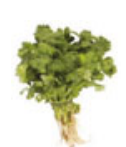

30

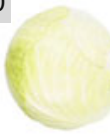

7

15

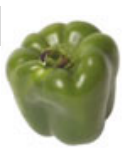

34

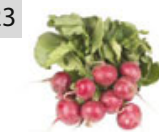

35

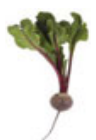

36

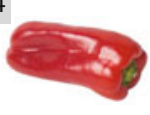

8

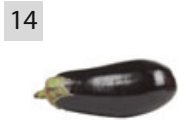

5
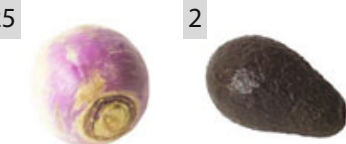

32
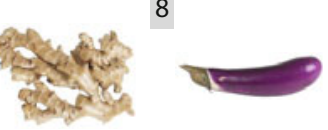

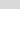

Figure 1. Mean rating results from Experiment 1 for the fruit (A) and vegetable (B) objects. The objects are in reading order by the mean of the average rating. The bar graph shows the range of size judgments for individual objects. For each bar, the means of the small, average, and large sizes are indicated by the bottom, middle, and top lines, respectively. The vertical lines correspond to the $25 \%-75 \%$ confidence interval across participants. 

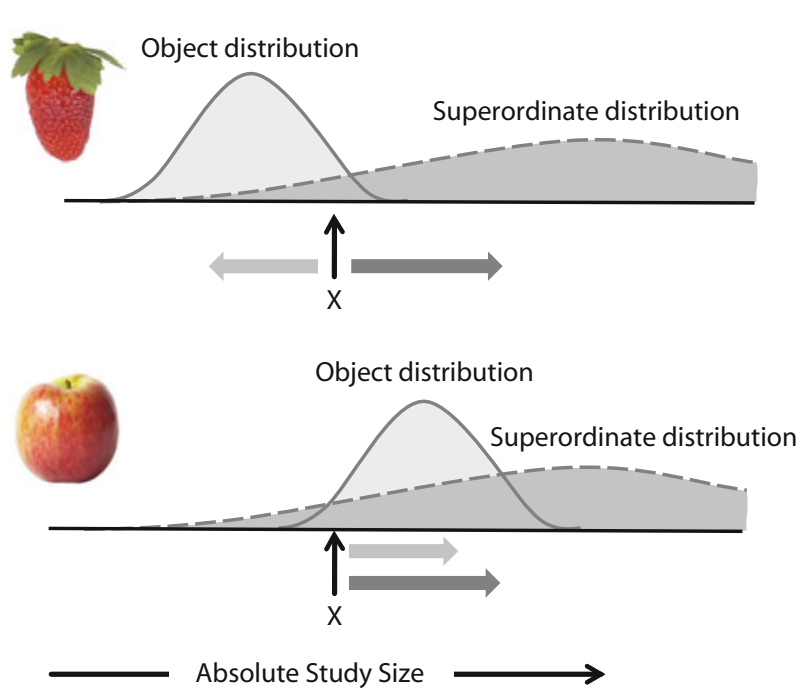

Figure 2. Predicted influences of prior knowledge at the object and superordinate levels. The distributions illustrate the hypothetical range of sizes associated with the fruit category and two particular objects: a strawberry and an apple. If the two objects are shown at the same study size, marked by "X," the strawberry and apple are presented at a very large and very small size, respectively, relative to their object distributions. This leads to object bias in opposite directions, toward the center of the respective object distributions, as shown by the light-colored arrows. In contrast, the superordinate bias operates in the same direction, toward the center of the fruit distribution, as shown by the darkcolored arrows.

study size. An object-level bias would lead to an underestimation for the strawberry and an overestimation for the apple toward the centers of their respective object size distributions. A superordinate-level bias would lead to an overestimation for both the apple and the strawberry. Because these effects might operate simultaneously, it is unclear what the combined result would be of the objectlevel and superordinate-level biases. However, the key prediction was that the objects that are studied at the same absolute study size can be differentially biased when the objects are associated with different object-level prior knowledge. This prediction also excludes the possibility that the bias is due to sequential effects or edge effects, whereby participants are reluctant to use the edges of the response scale.

In addition to the natural objects, we also tested recall for artificial shapes that have no natural size scale (see Figure 3) and presented these at the same sizes as the vegetable stimuli. The inclusion of such shapes allowed additional comparisons of the relative influences of prior knowledge for artificial and naturalistic stimuli. We expected that participants would not have any strong preexperimental expectations about size at either the object or the superordinate level. However, it was possible for participants to gain knowledge about the overall sizes of artificial shapes during the course of the experiment. Therefore, we hypothesized that recall would be biased toward the mean within the overall size distribution associated with the artificial shapes.

\section{Method}

Participants. There were 25 participants, undergraduate students at the University of California, Irvine. The participants were not involved in the norming study in Experiment 1 and were compensated with course credit.

Materials and Procedure. We used the 24 objects from the fruit and vegetable categories in Experiment 1. We also created a category of 24 artificial shapes, which are shown in Figure 3. These images were created by drawing outlines of objects and filling the interiors with a blue color. We employed a recall paradigm analogous to continuous recognition, in which study and test events are randomly interleaved. The images were blocked by category (fruits, vegetables, and shapes), and each of the 24 images within each category was studied and tested three times, for a total of 144 trials per block. Trials were presented in random order but were constrained such that all test images had been previously seen as a study image, and there was always an intervening test trial between each repeated study trial. Furthermore, the study size for a given image remained constant across the three repetitions. Each study image was presented for $2 \mathrm{sec}$ at the center of the right screen. The same comparison image that was used in Experiment 1 remained on the left screen at all times. Each test image was presented on the right screen, and the participants responded by clicking on one of two buttons on the screen: "Remember" or "Don't Remember." After this recognition judgment, the participants were then asked to scale the size of the test object using the mouse to move the slider presented on the right screen. The following question appeared with the slider: "What was the size of this object, compared to the object on the left, when you saw it at study?" Responses were measured on a scale of 0 to 1 . The participants were given clear verbal instructions in order to ensure that they understood the task and were shown real-life versions of the comparison object (a set of utensils) in order to ensure agreement on the frame of reference.

On study trials, the size of a fruit or vegetable object was selected from the ranges shown in Figure 1. For each object, the range extended from the mean of the small judgments to the mean of the large judgments. This range was divided into eight bins, separately for each object. Five percent of study sizes were drawn from Bins 1 and 8 (smallest and largest), 10\% from each of Bins 2 and 7, 15\% from each of Bins 3 and 6, and 20\% from each of Bins 4 and 5 (middle bins). This procedure ensured that the majority of the objects were presented at a size close to the mean size of the object and that few objects were shown at a size close to the smallest and largest acceptable sizes for that object. It also guaranteed that objects would never be presented outside their acceptable range. For the artificial shapes, the study sizes were sampled from the same distributions as for the vegetables. Each artificial shape was yoked to a vegetable object, such that the study size was the same as for the paired veg-

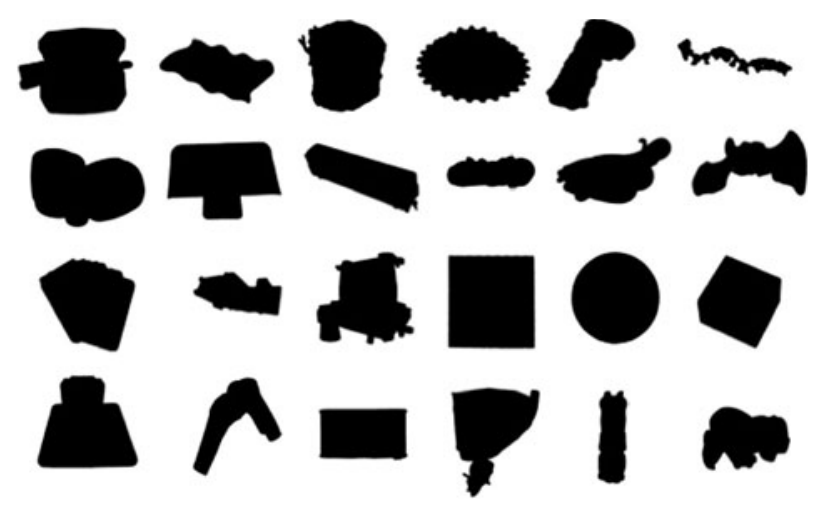

Figure 3. Artificial shapes used in Experiment 2. 
A

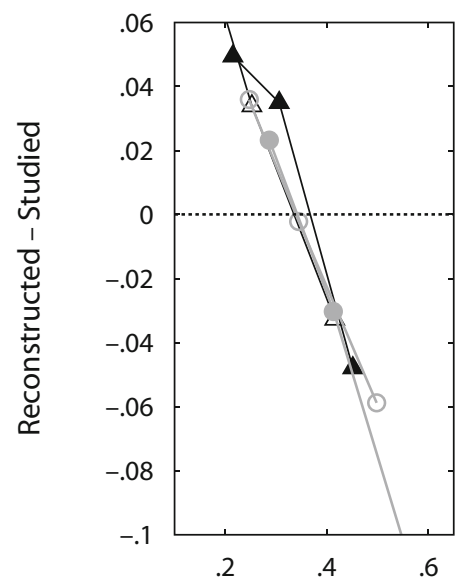

Absolute Study Size

\section{B}

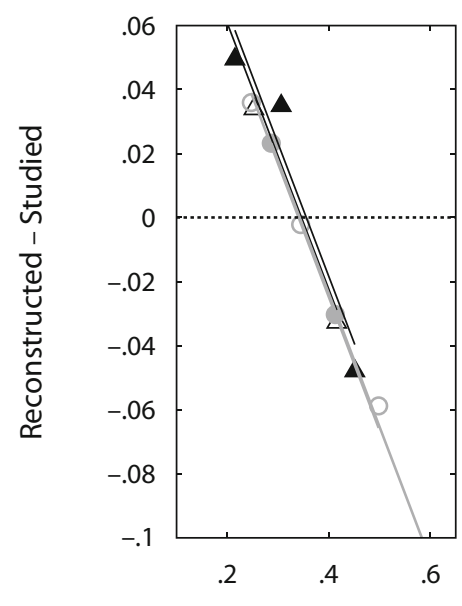

Absolute Study Size
Fruits

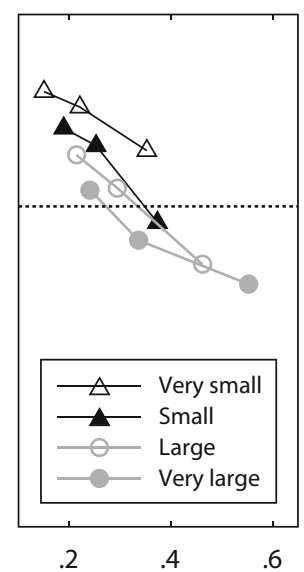

Absolute Study Size

Fruits

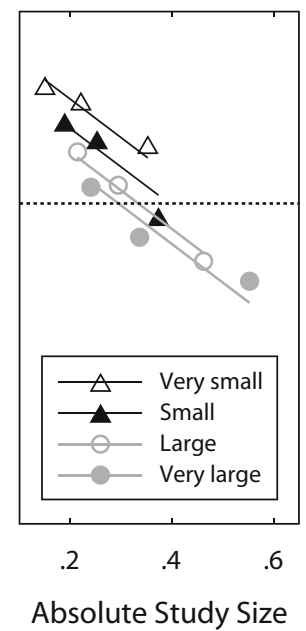

Vegetables

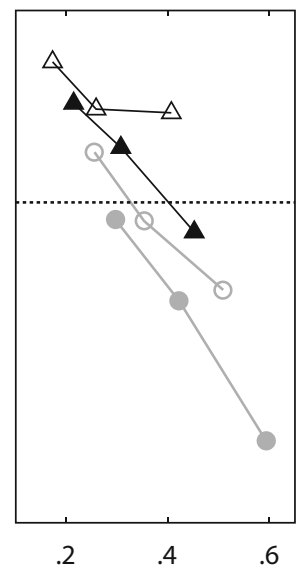

Absolute Study Size

Vegetables

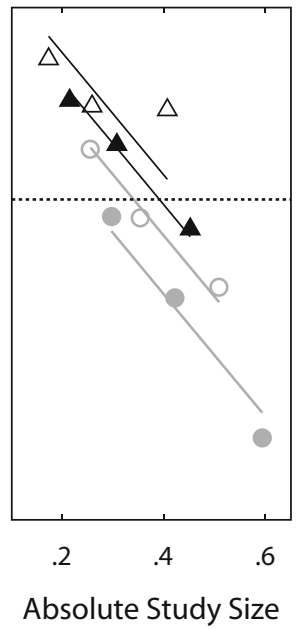

Figure 4. Recall biases as a function of absolute and relative study size. (A) Experimental data. Results are grouped by superordinate category and relative object size. (B) Linear regression model fits. Negative slopes are consistent with biases toward the center of the superordinate category range. Intercept differences are consistent with object-level knowledge effects.

etable. On test trials, the images were shown randomly at one of four sizes on the slider scale: $.2, .4, .6$, and .8. The slider was initiated at the corresponding location.

\section{Results}

To investigate the effect of prior knowledge at the object-category and superordinate-category levels, we measured recall error as the difference between the recalled size and the studied size. We restricted the analysis to hits only (i.e., remember responses). For visual clarity, we divided the study objects in the vegetable and fruit categories into four classes on the basis of the study size relative to the size ranges obtained in the norming study. We named the four classes very small, small, large, and very large. These sizes do not refer to the absolute size at which an object was studied but rather to the size relative to the distribution at the object level (e.g., the example in Figure 2 illustrates two objects presented at the same absolute study size but at different relative study sizes - a very small apple and a very large strawberry). Figure 4A shows the recall errors as a function of absolute study size for each superordinate category. The lines in the figure have two important aspects: slope and intercept. A negative slope indicates a bias to the center of the category range, such that small objects are overestimated (positive bias) and large objects are underestimated (negative bias). The difference between intercepts tests our key prediction that objects studied at the same absolute study size can be differentially biased, depending on prior knowledge at the object level. An intercept difference indicates a bias to the center of the object range, such that objects that are presented at a size 
Table 1

Mean Slopes and Intercepts by Category and Relative Object Size for Experiment 2

\begin{tabular}{|c|c|c|c|c|c|c|c|}
\hline & \multirow[b]{2}{*}{ Relative Object Size } & \multicolumn{2}{|c|}{ Fruits } & \multicolumn{2}{|c|}{ Vegetables } & \multicolumn{2}{|c|}{ Shapes } \\
\hline & & $M$ & $S D$ & $M$ & $S D$ & $M$ & $S D$ \\
\hline Slopes & & -.120 & .127 & -.191 & .169 & -.415 & .201 \\
\hline \multirow{4}{*}{ Intercepts } & Very small & .057 & .049 & .089 & .062 & .144 & .075 \\
\hline & Small & .048 & .045 & .075 & .058 & .148 & .072 \\
\hline & Large & .040 & .044 & .065 & .059 & .142 & .072 \\
\hline & Very large & .035 & .054 & .047 & .065 & .143 & .016 \\
\hline
\end{tabular}

Note $-N=25$

that is small relative to the object size distribution have a greater overestimation error than do objects presented at a relatively large size. In order to statistically assess these effects, a linear regression model was fitted separately at the individual subject level. The regression model contained five parameters for each category: four intercept parameters, corresponding to the four relative object classes, and a single slope parameter (see Figure 4B for fits of this regression model). Table 1 shows the mean estimated slopes and intercepts across superordinate categories and relative object sizes. Separate $t$ tests revealed that the slope for each category was significantly smaller than 0 [fruits, $t(24)=-4.714, p=.000$; vegetables, $t(24)=-5.657, p=.000$; shapes, $t(24)=-10.754$, $p=.000]$. This result is consistent with a superordinatelevel influence of prior knowledge. An object-level effect is revealed by the intercept differences between relative object sizes. A repeated measures ANOVA for the shape category found no effect of intercept $[F(3,72)=$ $0.453, p=.716]$. This lack of difference between the intercepts in the shape category indicates that there is no object-level effect for the objects for which the participants had no preexperimental knowledge. A 2 (category) $\times 4$ (intercept) repeated measures ANOVA was performed for the fruit and vegetable categories. There was no significant main effect of category $[F(1,24)=$ $3.927, p=.059]$, indicating that there was no difference between the fruit and vegetable categories. There was a significant main effect of intercept $[F(3,72)=14.359$, $p=.000]$. Contrasts revealed that the bias for relatively very small objects was significantly larger than that for relatively small objects $[F(1,24)=4.403, p=.037]$, as was the bias for relatively small versus that for relatively large objects $[F(1,24)=7.902, p=.010]$ and the bias for relatively large versus that for relatively very large objects $[F(1,24)=6.022, p=.022]$. These differences in intercepts confirm the prediction that objects studied at the same size are recalled differently depending on the relative size of the studied object. This is consistent with an object-level influence of prior knowledge. The shape category showed no such effect.

We also determined the relationship between recognition response (remember or don't remember) and recall performance. In this analysis, recall error was assessed by root-mean squared error (RMSE), which measures the absolute deviations between the studied and recalled sizes of the objects by participant. ${ }^{2}$ Larger RMSE values indicate worse performance. Figure 5 shows the RMSE by category and recognition response. A 2 (remember) $\times 3$ (category) repeated measures ANOVA was performed. There was a significant main effect of remembering $[F(1,6)=$ $10.712, p=.017]$. Recall error was significantly lower for hits (i.e., remember responses) than for misses (don't remember responses). There was a significant main effect of category $[F(2,12)=14.572, p=.001]$. Contrasts revealed that recall error was greater for artificial shapes than for fruits $[F(1,6)=32.155, p=.001]$ and for vegetables $[F(1,6)=8.571, p=.026]$. There was no significant difference between the recall errors for fruits and for vegetables $[F(1,6)=4.91, p=.069]$. The worst performance was observed for misses in the artificial shape category. In this condition, performance was similar to chance as measured by the mean of the size distribution of the artificial shapes (indicated in Figure 5 by the dotted line). Note that this distribution was by design identical to the vegetable size distribution. There was a significant interaction effect between remember responses and category $[F(2,12)=$ 4.721, $p=.031]$. Even when participants indicated that they had no conscious recollection of seeing the object at

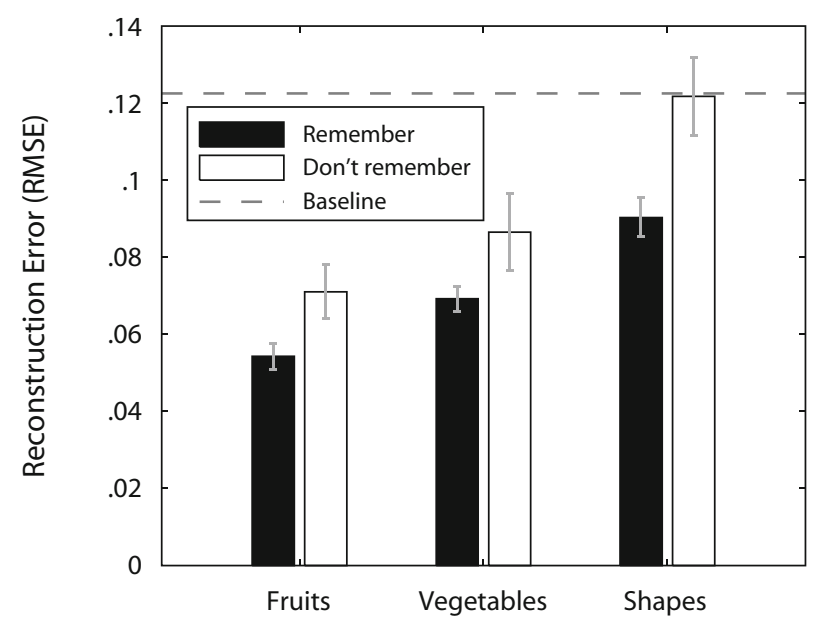

Figure 5. Recall error in Experiment 2 measured by root-mean squared error (RMSE). The error is grouped by superordinate category and by recognition response. The baseline error is equivalent to responses based on the mean of the vegetable sizes or, equivalently, the shapes category. Error bars indicate standard errors of the means. 
study, they performed better in both the fruit and the vegetable categories than in the artificial shape category.

\section{DISCUSSION}

We have shown that episodic memories are influenced by prior knowledge and that such influences appear to be hierarchically structured. Objects above the superordinate category mean (e.g., a pineapple) were reconstructed smaller, and objects below the superordinate category mean (e.g., a raspberry) were reconstructed larger. At the same time, an object studied at the small end of that object range (e.g., a small apple) was overestimated, whereas an object studied at the large end of that range (e.g., a large apple) was underestimated, independent of the absolute study size. The biases in size recall are consistent with prior knowledge exerting an influence at both the object-category and the superordinatecategory levels.

Our experimental design allowed us to rule out various alternative hypotheses about the object-level effects. Sailor and Antoine (2005) hypothesized that temporal contiguity (i.e., sequential effects) produced the center bias effects of the Huttenlocher et al. (1991; Huttenlocher et al., 2000) studies and that reconstruction did not vary systematically as a function of category membership. We, however, have demonstrated that not only does reconstruction vary systematically as a function of category membership but also that these findings cannot be due to sequential effects. Sequential effects cannot be responsible for the object-level effects, because we have demonstrated that objects studied at the exact same size can be recalled differently, depending on the prior knowledge associated with the object. Our findings also preclude the possibility that the object-level bias is due to edge effects and slider start location. These effects would not predict differential bias between vegetables and shapes that are studied at the same size, and they are unable to explain the object-level effects. Therefore, our results show that prior knowledge exerts strong effects on long-term memory and that prior knowledge corrects for noisy episodic memories at the object level. On the other hand, it is not entirely clear whether the negative slopes in Experiment 2 can be solely attributed to prior knowledge at the superordinate-category level. It is possible that additional factors have contributed to this effect. For example, sequential effects can lead to a bias toward the center of the overall size distribution. Similarly, it is not clear whether the participants were utilizing prior knowledge at the superordinate level (e.g., fruits) or at an even more abstract level encompassing all objects encountered during the experiment.

Overall, our results are consistent with the view that the interaction between episodic memory and prior knowledge occurs at multiple levels of abstraction. The nature of the combination of different sources of prior knowledge might depend on the familiarity of the object. Very specific prior knowledge associated with the object might be utilized during recall, when the participant is very familiar with the object. On the other hand, if the object is unfamiliar, prior knowledge at a higher level of abstraction (such as the superordinate level) might be engaged.

In contrast to previous studies, our experimental paradigm allowed us to disentangle recall error for objects that were or were not recognized. One striking finding was that even in the absence of recognition, a smaller reconstruction error was found for objects associated with preexperimental knowledge (fruits and vegetables) than for objects without such knowledge (shapes). This difference cannot be attributed to differences in the size distribution, since the shapes and vegetables were studied at the exact same sizes. In the absence of episodic information about a studied artificial shape, the lowest recall error (as assessed by RMSE) is achieved by responding with the mean of the superordinate category distribution observed in the experiment. The participants appeared to have followed this strategy (see Figure 4). However, in the absence of episodic information about a studied vegetable or fruit, a lower error can be achieved by using both the superordinate and the object size distributions to generate a response. Such knowledge about sizes of specific objects could only have been attained outside the experiment, because in the experimental context, the participants saw a particular object only at a single size. This result supports findings by Huttenlocher et al. (1991; Huttenlocher et al., 2000) that prior knowledge can serve a useful role to improve average performance in recall when episodic memory is weak, incomplete, or error prone.

Using prior knowledge at multiple levels of abstraction is an efficient strategy that allows generalization over experiences and correction of noisy memories. Bartlett's (1932) finding that his British participants tended to recall the natives sailing in a boat rather than a canoe is in line with the predictions of a hierarchical effect that recall for an unfamiliar object, such as a canoe, is influenced by a higher, more abstract level of knowledge - namely, that people sail in boats. In essence, the participants had weak memory traces when recalling the story months or even years after initial exposure but were able to use prior knowledge at a higher level of abstraction to aid recall.

\section{AUTHOR NOTE}

The authors thank Ken Malmberg and Scott Brown for discussions that helped shape this research. Correspondence concerning this article should be addressed to P. Hemmer, Department of Cognitive Sciences, University of California, Irvine, 3151 Social Sciences Plaza, Irvine, CA 92697-5100 (e-mail: phemmer@uci.edu).

\section{REFERENCES}

Bartlett, F. C. (1932). Remembering: A study in experimental and social psychology. Cambridge: Cambridge University Press.

Biernat, M. (1993). Gender and height: Developmental patterns in knowledge and use of an accurate stereotype. Sex Roles, 29, 691-713.

Bower, G. H., Karlin, M. B., \& Dueck, A. (1975). Comprehension and memory for pictures. Memory \& Cognition, 3, 216-220.

Bransford, J. D., \& Johnson, M. K. (1972). Contextual prerequisites 
for understanding: Some investigations of comprehension and recall. Journal of Verbal Learning \& Verbal Behavior, 11, 717-726.

Brewer, W. F., \& Treyens, J. C. (1981). Role of schemata in memory for places. Cognitive Psychology, 13, 207-230.

Crawford, E. L., Huttenlocher, J., \& Engebretson, P. H. (2000). Category effects on estimates of stimuli: Perception or reconstruction? Psychological Science, 11, 280-284.

Huttenlocher, J., Hedges, L. V., \& Duncan, S. (1991). Categories and particulars: Prototype effects in estimating spatial location. Psychological Review, 98, 352-376.

Huttenlocher, J., Hedges, L. V., \& Vevea, J. (2000). Why do categories affect stimulus judgment? Journal of Experimental Psychology: General, 129, 220-241.

Kerst, S. M., \& Howard, J. H., JR. (1978). Memory psychophysics for visual area and length. Memory \& Cognition, 6, 327-335.

Moyer, R. S., Bradley, D. R., Sorensen, M. H., Whiting, C., \& MANSFIELD, D. P. (1978). Psychophysical functions for perceived and remembered size. Science, 200, 330-332.

Rehnman, J., \& Herlitz, A. (2006). Higher face recognition ability in girls: Magnified by own-sex and own-ethnicity bias. Memory, 14, 289-296.

Roediger, H. L., III, \& McDermott, K. B. (1995). Creating false memories: Remembering words not presented in lists. Journal of Experimental Psychology: Learning, Memory, \& Cognition, 21, 803-814.

SAILOR, K. M., \& ANToINE, M. (2005). Is memory for stimulus magnitude Bayesian? Memory \& Cognition, 33, 840-851.

\section{NOTES}

1. Data from 4 participants were discarded because these participants made large judgments that were smaller than their small judgments (or vice versa), indicating that they did not understand the task.

2. RMSE $=\sqrt{\sum_{i}\left(x_{i}^{\text {recall }}-x_{i}^{\text {study }}\right)^{2} / N}$, where $x^{\text {recall }}$ and $x^{\text {study }}$ are the recalled and studied sizes, respectively, and $N$ is the number of objects in the calculation.

(Manuscript received May 5, 2008;

revision accepted for publication August 5, 2008.) 